TRANSACTIONS OF THE

AMERICAN MATHEMATICAL SOCIETY

Volume 356, Number 9, Pages 3739-3750

S 0002-9947(03)03529-3

Article electronically published on December 15, 2003

\title{
NON-ISOTOPIC SYMPLECTIC TORI IN THE SAME HOMOLOGY CLASS
}

\author{
TOLGA ETGÜ AND B. DOUG PARK
}

\begin{abstract}
For any pair of integers $n \geq 1$ and $q \geq 2$, we construct an infinite family of mutually non-isotopic symplectic tori representing the homology class $q[F]$ of an elliptic surface $E(n)$, where $[F]$ is the homology class of the fiber. We also show how such families can be non-isotopically and symplectically embedded into a more general class of symplectic 4-manifolds.
\end{abstract}

\section{INTRODUCTION}

In a fixed 2-dimensional homology class of a complex surface, there are at most finitely many complex curves up to smooth isotopy. In contrast, there exist examples of homology classes in symplectic 4-manifolds that are represented by infinitely many non-isotopic connected symplectic surfaces. In [FS3 Fintushel and Stern proved that $q[F] \in H_{2}(X ; \mathbb{Z})$ is such a class provided that $q \geq 4$ is an even integer and $F$ is a symplectic $c$-embedded torus, i.e., a homologically non-trivial torus of self-intersection 0 with first homology generated by two circles which bound disks of self-intersection -1 in $X$, in a simply-connected symplectic 4-manifold $X$ (e.g. we can take $X$ to be an elliptic surface $E(n)$ and $F$ to be a regular fiber). In this paper we generalize their result by constructing infinitely many non-isotopic symplectic tori in $q[F] \in H_{2}(X ; \mathbb{Z})$ for each $q \geq 3$ (even or odd) in the case when $F$ is a symplectic torus in a symplectic 4 -manifold $X$ satisfying $[F]^{2}=0$ and $H^{1}(X \backslash \nu(F) ; \mathbb{Z})=0$. Moreover, as a consequence of our calculation, it will be apparent that no two of these tori are equivalent under the action of $\operatorname{Diff}(X)$, the group of self-diffeomorphisms of $X$. In some special cases our result can be slightly improved to include $2[F]$ as in the following theorem.

Theorem 1. For any pair of integers $n \geq 1$ and $q \geq 2$, there exists an infinite family of mutually non-isotopic symplectic tori representing the homology class $q[F]$ of an elliptic surface $E(n)$, where $[F]$ is the homology class of the fiber.

It should be noted that in [V2], Vidussi has constructed such families for every positive multiple of the fiber class in $E(n)$ provided that $n \geq 3$. However, it still remains an interesting open problem to construct infinite families of non-isotopic symplectic tori in the fiber class $[F]$ of $E(1)$ and $E(2)$. We should also note that our construction can be carried out for a far more general class of 4-manifolds than those in [V2].

Received by the editors December 13, 2002 and, in revised form, June 6, 2003.

2000 Mathematics Subject Classification. Primary 57R17, 57R57; Secondary 53D35, 57R95.

The second author was partially supported by an NSERC research grant.

(C)2003 American Mathematical Society 
In all of the above examples, families of braids are used to construct symplectic tori, and Seiberg-Witten theory is used to distinguish them. The family of braids we use in this paper are, in some sense, the simplest possible and this allowed us not only to calculate the Seiberg-Witten invariants of the corresponding 4-manifolds completely, but also to reprove and extend the results in [FS3] and V2] (except for the case of fiber class itself in $E(n)$ for $n \geq 3$ ). From our construction, it will be apparent that any other collection of reasonably complicated braids will also give rise to many more new examples of non-isotopic symplectic tori.

In the next section we construct a family of tori, $\left\{T_{p, q}\right\}_{p, q \geq 2}$, in $T^{2} \times D^{2}$ which could be identified with a regular tubular neighborhood of a torus of self-intersection 0 in a 4-manifold. Then we show how these tori could be symplectically embedded in $E(n)$. In Section 3 we calculate the Seiberg-Witten invariants of symplectic 4manifolds obtained as the fiber sum of $E(n)$ and $E(r)$ along $T_{p, q} \subset E(n)$ and a fiber in $E(r)$. As a consequence of these computations we prove Theorem 1 In Section 4 we explain how Theorem 1 could be generalized to a larger class of 4-manifolds (see Theorem 14).

\section{BRAID CONSTRUCTION}

We first construct an infinite family of non-isotopic symplectic tori $\left\{T_{p, q}\right\}_{p, q \geq 2}$ representing $q[F]$ in $E(1)$, and show that these remain non-isotopic after fibersumming with $E(n-1)$. That is, we will construct an infinite family of tori that embed non-isotopically into $E(n)$ for any $n \geq 1$.

Even though we are unable to show that $T_{1, q}$ is not isotopic to any torus in $\left\{T_{p, q}\right\}_{p \geq 2}$ in general (for fixed $q$ ), we will still include it in our construction and computations for the sake of completeness. For any pair of integers $p \geq 1$ and $q \geq 2$, consider the $q$-strand braid $B_{p, q}$ in Figure 1 . The closed braid $\hat{B}_{p, q}$ is a $(2,2 p-1)$-torus knot, and is the "simplest" knot in $S^{3}$ with $(2 p-1)$ crossings, which is usually denoted by $(2 p-1)_{1}$ in the literature (see e.g. [BZ] or [Ro]).

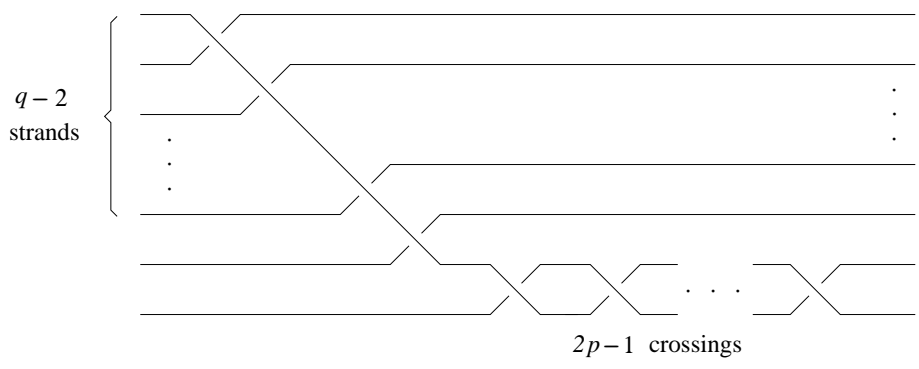

Figure 1. Braid $B_{p, q}$ for $p \geq 1$ and $q \geq 2$

Let $L_{p, q}$ denote the two-component link in $S^{3}$ which is the union of the closed braid $\hat{B}_{p, q}$ and its axis $A$. See Figure 2 for a picture of $L_{2,4}$.

Next we review the generalization of the link surgery construction of Fintushel and Stern [FS2] by Vidussi [V1]. For an $m$-component link $L$, choose a homology basis of simple curves $\left\{\alpha_{i}, \beta_{i}\right\}_{i=1}^{m}$ such that the pair $\left\{\alpha_{i}, \beta_{i}\right\}$ lies in the $i$ th boundary component of the link exterior and the intersection of $\alpha_{i}$ and $\beta_{i}$ is 1 . We define the 


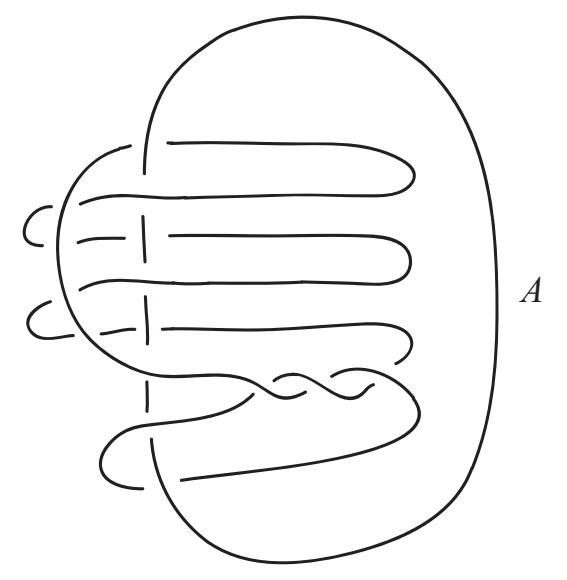

Figure 2. Two-component link $L_{2,4}$

link surgery manifold

$$
E\left(n_{1}, \ldots, n_{m}\right)_{L}:=\left[\coprod_{i=1}^{m} E\left(n_{i}\right) \backslash \nu F_{i}\right]_{F_{i} \times \partial D^{2}=\left(S^{1} \times \alpha_{i}\right) \times \beta_{i}}\left[S^{1} \times\left(S^{3} \backslash \nu L\right)\right],
$$

where $\nu$ denotes the tubular neighborhoods. Here, the gluing diffeomorphisms between the boundary 3-tori identify the torus fiber $F_{i}$ of $E\left(n_{i}\right)$ with $S^{1} \times \alpha_{i}$, and act as complex conjugation on the last remaining $S^{1}$ factor. Strictly speaking, our gluing construction depends on our choice of basis $\left\{\alpha_{i}, \beta_{i}\right\}_{i=1}^{m}$, but we will suppress this dependence in our notation. (See also Remark 10)

As before let $A$ denote the unknot which is the axis of the closed braid $\hat{B}_{p, q}$. Choose the homology basis $\left(\alpha_{1}, \beta_{1}\right)=(\mu(A), \lambda(A))$ for the complement $\left(S^{3} \backslash \nu A\right)$, where $\mu$ and $\lambda$ denote the meridian and longitude, respectively.

Lemma 2. $E(1)_{A}=E(1)$.

Proof. Note that the exterior of the unknot $\left(S^{3} \backslash \nu A\right)$ is diffeomorphic to a solid torus $S^{1} \times D^{2}$. Hence there is a diffeomorphism between the tubular neighborhood of a regular fiber $\nu F=T^{2} \times D^{2}$ and the Cartesian product $\left[S^{1} \times\left(S^{3} \backslash \nu A\right)\right]$.

Now consider $\hat{B}_{p, q}$ as a knot inside the solid torus $\left(S^{3} \backslash \nu A\right)$. Define $T_{p, q}:=$ $S^{1} \times \hat{B}_{p, q}$, which is a torus embedded in $\left[S^{1} \times\left(S^{3} \backslash \nu A\right)\right] \approx \nu F \subset E(1)_{A}$.

Lemma 3. The torus $T_{p, q} \subset E(1)_{A}$ is a symplectic submanifold and represents the homology class $q[F]$.

Proof. First note that $\hat{B}_{p, q}$ is homologous to $q \mu(A)$ in $H_{1}\left(S^{3} \backslash \nu A\right)$. Since the fiber $F$ is identified with $S^{1} \times \mu(A)$ in $E(1)_{A}$, the torus $T_{p, q}$ is homologous to $q F$ in $E(1)_{A}=E(1)$. To show that $T_{p, q}$ is symplectic, we can proceed as in [FS3] and V2. The key step is once again verifying that the $\operatorname{knot} \hat{B}_{p, q} \subset\left(S^{3} \backslash \nu A\right) \approx\left(S^{1} \times D^{2}\right)$ is transverse to the disks $\{$ pt. $\} \times D^{2}$.

Next we show that the torus $T_{p, q}$ can be embedded in $E(n)$ for any $n \geq 1$. For $n \geq 2$, we can write $E(n)$ as the fiber sum,

(1) $E(n)=E(1)_{A} \#_{F_{1}^{\prime}=F_{2}} E(n-1)=\left[E(1)_{A} \backslash \nu F_{1}^{\prime}\right]_{F_{1}^{\prime} \times \partial D^{2}=F_{2} \times \partial D^{2}}\left[E(n-1) \backslash \nu F_{2}\right]$, 
where $F_{1}^{\prime}$ is a regular torus fiber lying outside $\left[S^{1} \times\left(S^{3} \backslash \nu A\right)\right]$. We immediately see that $T_{p, q}$ can be embedded into the first fiber summand and hence into $E(n)$. Without causing too much confusion, we will continue to denote this embedded torus in $E(n)$ by $T_{p, q}$. Note that Lemma 3 continues to hold for the torus $T_{p, q} \subset$ $E(n)$.

Since the torus $T_{p, q} \subset E(n)$ has self-intersection zero, we can form the (generalized) fiber sum,

$$
E(n) \#_{T_{p, q}=F} E(r):=\left[E(n) \backslash \nu T_{p, q}\right] \bigcup_{T_{p, q} \times \partial D^{2}=F \times \partial D^{2}}[E(r) \backslash \nu F] .
$$

The gluing diffeomorphism $\varphi$ identifies $T_{p, q}$ with $F$ and acts as complex conjugation on the $\partial D^{2}$ factor. The torus fiber $F$ in $E(r)$ has a canonical framing coming from the elliptic fibration. Note that when $n \geq 2$ there is a canonical framing of $T_{p, q}$ in $E(n)$ that is inherited from a fixed framing of $T_{p, q}$ in $E(1)$ by our fiber sum description (1).

Lemma 4. The diffeomorphism type of the fiber sum $E(n) \#_{T_{p, q}=F} E(1)$ does not depend on the chosen framing of $T_{p, q}$.

Proof. Changing the framing of $T_{p, q}$ amounts to composing the gluing map $\varphi$ with an orientation-preserving self-diffeomorphism of $\partial \nu F \cong T^{3}$. However, Matumoto has shown in $\mathrm{Ma}$ that any orientation-preserving self-diffeomorphism of $\partial \nu F$ extends over $[E(1) \backslash \nu F]$.

We remark that the gluing formula in $\mathrm{Pa}$ cannot be used to compute the Seiberg-Witten invariant of the fiber sum (2), since the triple $\left(\left[E(n) \backslash \nu T_{p, q}\right]\right.$, $[E(r) \backslash \nu F], \varphi)$ is not admissible in the sense of Definition 8 in $[\mathrm{Pa}]$. However, the key observation is that this fiber sum can be expressed as a link surgery manifold.

Lemma 5. $E(n) \#_{T_{p, q}=F} E(r)=E(n, r)_{L_{p, q}}$.

Proof. For the construction of $E(n, r)_{L_{p, q}}$, we choose the homology basis $\left(\alpha_{1}, \beta_{1}\right)=$ $(\mu(A), \lambda(A))$, and $\left(\alpha_{2}, \beta_{2}\right)=\left(\lambda\left(\hat{B}_{p, q}\right),-\mu\left(\hat{B}_{p, q}\right)\right)$. The identification with the fiber sum is now immediate.

\section{Seiberg-Witten invariants}

To prove Theorem 1, we need to show that the family of tori $\left\{T_{p, q}\right\}_{p \geq 2}$ we constructed are mutually non-isotopic for fixed $q$. It is enough to show that the corresponding family of 4-manifolds $\left\{E(n, 1)_{L_{p, q}}\right\}_{p \geq 2}$ are mutually non-diffeomorphic, since any isotopy between the tori $\left\{T_{p, q}\right\}$ will translate into diffeomorphism between corresponding 4-manifolds $\left\{E(n, 1)_{L_{p, q}}\right\}$. We shall compute their Seiberg-Witten invariants to show that $\left\{E(n, 1)_{L_{p, q}}\right\}$ are mutually non-diffeomorphic.

Let $\Delta_{L_{p, q}}(x, t)$ denote the Alexander polynomial of the link $L_{p, q}$, where the variable $x$ corresponds to the axis $A$.

Lemma 6. $\Delta_{L_{p, 2}}(x, t)=1+x t^{2 p-1}$, and when $q \geq 3$,

$$
\Delta_{L_{p, q}}(x, t)=1+x^{q-1} t^{2 p+q-3}+x t^{2}\left(\frac{1+t^{2 p-3}}{1+t}\right)\left(\frac{1-(x t)^{q-2}}{1-x t}\right) .
$$


Proof. The braid group on $q$ strands is generated by the elementary braid transpositions $\sigma_{1}, \ldots, \sigma_{q-1}$, where $\sigma_{i}$ denotes the crossing of the $(i+1)$ st strand over the $i$ th. Note that

$$
B_{p, q}=\sigma_{q-1} \sigma_{q-2} \cdots \sigma_{2} \sigma_{1}^{2 p-1} .
$$

By Theorem 1 in [Mo], we have

$$
\Delta_{L_{p, q}}(x, t)=\operatorname{det}\left(I-x C_{q-1}^{(q-1)} C_{q-2}^{(q-1)} \cdots C_{2}^{(q-1)}\left(C_{1}^{(q-1)}\right)^{2 p-1}\right),
$$

where $C_{i}^{(q-1)}$ denotes the following $(q-1) \times(q-1)$ matrix which differs from the identity matrix $I$ only in the three places shown on the $i$ th row:

$$
C_{i}^{(q-1)}:=\left(\begin{array}{cccccccc}
1 & & & & & & \\
& \ddots & & & & & \\
& & 1 & & & & \\
& & t & -t & 1 & & \\
& & & & 1 & & \\
& & & & & \ddots & \\
& & & & & & 1
\end{array}\right) .
$$

When $i=1$ or $i=q-1$, the matrix is truncated appropriately to give two non-zero entries in row $i$.

The statement for $q=2$ case is obvious. We are going to prove the case $q \geq 3$ by induction on $q$. The main step of this induction is proving that $D_{p, q}=x t D_{p, q-1}$, where $D_{p, q}:=\Delta_{L_{p, q+1}}(x, t)-\Delta_{L_{p, q}}(x, t)$. Then we prove that $\Delta_{L_{p, 3}}(x, t)=1+$ $x^{2} t^{2 p}+x t^{2}\left(\frac{1+t^{2 p-3}}{1+t}\right)$ and this implies $D_{p, 2}=x t^{2}\left(1-t+t^{2}-\cdots-t^{2 p-3}\right)+x^{2} t^{2 p}$ and hence $D_{p, q}=x^{q-1} t^{q}\left(1-t+t^{2}-\cdots-t^{2 p-3}\right)+x^{q} t^{2 p+q-2}$. Finally, by using the induction assumption and $\Delta_{L_{p, q+1}}(x, t)=\Delta_{L_{p, q}}(x, t)+D_{p, q}$ we finish the proof of the lemma.

$\Delta_{L_{p, q+1}}(x, t)=\operatorname{det}\left(I-x \Gamma_{p, q+1}\right)$, where $\Gamma_{p, q+1}:=C_{q}^{(q)} C_{q-1}^{(q)} \cdots C_{2}^{(q)}\left(C_{1}^{(q)}\right)^{2 p-1}$. Note that

$$
C_{i}^{(q)}=\left(\begin{array}{cccc} 
& & & 0 \\
& C_{i}^{(q-1)} & & \vdots \\
& & & 0 \\
0 & \cdots & 0 & 1
\end{array}\right)
$$

for $i \in\{1,2, \ldots, q-2\}$, so we must have

$$
\begin{aligned}
& \Gamma_{p, q+1}=C_{q}^{(q)} C_{q-1}^{(q)}\left(\begin{array}{cccc} 
& & & 0 \\
& C_{q-1}^{(q-1)} & & \vdots \\
0 & \ldots & 0 & 1
\end{array}\right)^{-1}\left(\begin{array}{cccc} 
& & \\
& & & \\
& \Gamma_{p, q} & & \vdots \\
& & & 0 \\
0 & \ldots & 0 & 1
\end{array}\right) \\
& =\left(\begin{array}{cccc}
1 & & & \\
& \ddots & & \\
& & 1 & 1 \\
& & t & 0
\end{array}\right)\left(\begin{array}{cccc} 
& & & 0 \\
& \Gamma_{p, q} & & \vdots \\
& & & 0 \\
0 & \ldots & 0 & 1
\end{array}\right) .
\end{aligned}
$$


Hence, it follows that

$$
\Gamma_{p, q+1}=\left(\begin{array}{cc} 
& 0 \\
\Gamma_{p, q} & \vdots \\
& 0 \\
t\left(\Gamma_{p, q}\right)_{(q-1, *)} & 0
\end{array}\right)
$$

and

$$
I-x \Gamma_{p, q+1}=\left(\begin{array}{cc} 
& 0 \\
I-x \Gamma_{p, q} & \vdots \\
& 0 \\
-x t\left(\Gamma_{p, q}\right)_{(q-1, *)} & 1
\end{array}\right),
$$

where $\left(\Gamma_{p, q}\right)_{(q-1, *)}$ denotes the last row of $\Gamma_{p, q}$. When we calculate the determinant of the matrix $I-x \Gamma_{p, q+1}$ by expanding along its last column we get the following equality:

$$
\operatorname{det}\left(I-x \Gamma_{p, q+1}\right)=\operatorname{det}\left(I-x \Gamma_{p, q}\right)-(-x) t\left[\operatorname{det}\left(I-x \Gamma_{p, q}\right)-\operatorname{det}\left(I-x \Gamma_{p, q-1}\right)\right] .
$$

To prove the above equality, observe that all but the last row of the minor of the matrix $I-x \Gamma_{p, q+1}$ corresponding to the entry $-x$ in the last column are the same as the rows of $I-x \Gamma_{p, q}$, and the last row of the minor is $t$ times the last row of $I-x \Gamma_{p, q}$ except for the last entry. In the minor, this entry is 0 , whereas in $I-x \Gamma_{p, q}$ this entry is 1 (since (4) shows that the last diagonal entry of $\Gamma_{p, q}$ is 0)1 1 This observation is why the determinant of the minor corresponding to $-x$ is $t$ times the difference between the determinant of $I-x \Gamma_{p, q}$ and the determinant of the minor of $I-x \Gamma_{p, q}$ obtained by deleting the last row and the last column (and this minor is nothing but $\left.I-x \Gamma_{p, q-1}\right)$.

$D_{p, q}=x t D_{p, q-1}$ now follows trivially from (5). The next step is to calculate $\Delta_{L_{p, 3}}(x, t)$. By (3), we have

$$
\Delta_{L_{p, 3}}(x, t)=\operatorname{det}\left[\left(\begin{array}{ll}
1 & 0 \\
0 & 1
\end{array}\right)-x\left(\begin{array}{cc}
1 & 0 \\
t & -t
\end{array}\right)\left(\begin{array}{cc}
-t & 1 \\
0 & 1
\end{array}\right)^{2 p-1}\right] .
$$

An easy induction argument shows that

$$
\left(\begin{array}{cc}
-t & 1 \\
0 & 1
\end{array}\right)^{2 p-1}=\left(\begin{array}{cc}
-t^{2 p-1} & \frac{1+t^{2 p-1}}{1+t} \\
0 & 1
\end{array}\right)
$$

and then an explicit calculation gives

$$
\Delta_{L_{p, 3}}(x, t)=1+x^{2} t^{2 p}+x t^{2}\left(\frac{1+t^{2 p-3}}{1+t}\right) .
$$

\footnotetext{
${ }^{1}$ Strictly speaking, the last diagonal entry of $\Gamma_{p, q}$ is 0 , only if $q \geq 4$. Nevertheless, for $q=3$, (5) could be confirmed by a direct calculation of $\Delta_{L_{p, 4}}(x, t)$ along the same lines as the calculation of $\Delta_{L_{p, 3}}(x, t)$.
} 
As we discussed before, to finish the proof one only needs to check that

$$
\begin{gathered}
1+x^{q-1} t^{2 p+q-3}+x t^{2}\left(\frac{1+t^{2 p-3}}{1+t}\right)\left(\frac{1-(x t)^{q-2}}{1-x t}\right) \\
+x^{q-1} t^{q}\left(1-t+t^{2}-\cdots-t^{2 p-3}\right)+x^{q} t^{2 p+q-2} \\
=1+x^{q} t^{2 p+q-2}+x t^{2}\left(\frac{1+t^{2 p-3}}{1+t}\right)\left(\frac{1-(x t)^{q-1}}{1-x t}\right) .
\end{gathered}
$$

Recall that the Seiberg-Witten invariant $\overline{S W_{X}}$ of a 4-manifold $X$ (with $b_{2}^{+}(X)>$ 1) can be thought of as an element of the group ring of $H_{2}(X ; \mathbb{Z})$, i.e., $\overline{S W}_{X} \in$ $\mathbb{Z}\left[H_{2}(X ; \mathbb{Z})\right]$. If we write $\overline{S W}_{X}=\sum_{g} a_{g} g$, then we say that $g \in H_{2}(X ; \mathbb{Z})$ is a Seiberg-Witten basic class of $X$ if $a_{g} \neq 0$.

Lemma 7. Let $\iota:\left[S^{1} \times\left(S^{3} \backslash \nu L_{p, q}\right)\right] \rightarrow E(n, r)_{L_{p, q}}$ be the inclusion map. Let $\xi:=\iota_{*}\left[S^{1} \times \mu(A)\right], \tau:=\iota_{*}\left[S^{1} \times \mu\left(\hat{B}_{p, q}\right)\right] \in H_{2}\left(E(n, r)_{L_{p, q}} ; \mathbb{Z}\right)$. If $p \geq 2$ and $q \geq 3$, then the Seiberg-Witten invariant of $E(n, r)_{L_{p, q}}$ is

$$
\begin{aligned}
\overline{S W}_{E(n, r)_{L_{p, q}}} & =\left(\xi^{-1}-\xi\right)^{n-1}\left(\xi^{-q}-\xi^{q}\right)^{r-1}\left[\xi^{-(q-1)} \tau^{-(2 p+q-3)}+\xi^{q-1} \tau^{2 p+q-3}\right. \\
& \left.+\left(\tau^{-(2 p-4)} \sum_{i=0}^{2 p-4}\left(-\tau^{2}\right)^{i}\right)\left(\sum_{j=0}^{q-3}(\xi \tau)^{-(q-3)+2 j}\right)\right] .
\end{aligned}
$$

When $p=1$ and $q \geq 2$, we have

$$
\overline{S W}_{E(n, r)_{L_{1, q}}}=\left(\xi^{-1}-\xi\right)^{n-1}\left(\xi^{-q}-\xi^{q}\right)^{r-1}\left[\sum_{j=-1}^{q-2}(\xi \tau)^{-(q-3)+2 j}\right] .
$$

When $q=2$ and $p \geq 1$, we have

$$
\overline{S W}_{E(n, r)_{L_{p, 2}}}=\left(\xi^{-1}-\xi\right)^{n-1}\left(\xi^{-2}-\xi^{2}\right)^{r-1}\left[\xi^{-1} \tau^{-(2 p-1)}+\xi \tau^{2 p-1}\right] .
$$

Proof. Recall from [FS1] that $\overline{S W}_{E(n)}=\left([F]^{-1}-[F]\right)^{n-2}$. From the formulas in $\mathrm{Pa}$ and $[\mathrm{T} 2$, we know that

$$
\overline{S W}_{E(n) \backslash \nu F}=\left([F]^{-1}-[F]\right) \cdot \overline{S W}_{E(n)}=\left([F]^{-1}-[F]\right)^{n-1} .
$$

From the formulas in [FS2] and [T2], we conclude that

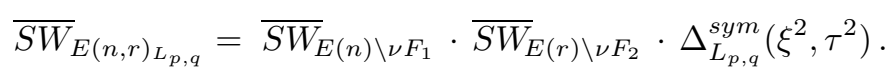

We also need to identify $\left[F_{1}\right]$ with $\xi$ and $\left[F_{2}\right]$ with $\xi^{q}$. This last identification is really necessary since we specifically chose to have $\alpha_{2}=\lambda\left(\hat{B}_{p, q}\right)=q \mu(A)=$ $q \alpha_{1} \in H_{1}\left(S^{3} \backslash \nu L_{p, q}\right)$ in the proof of Lemma 5. Hence we must have $\left[S^{1} \times \alpha_{2}\right]=$ $q\left[S^{1} \times \alpha_{1}\right] \in H_{2}\left(S^{1} \times\left(S^{3} \backslash \nu L_{p, q}\right)\right)$. Finally, we note that

$$
\Delta_{L_{p, q}}^{s y m}(x, t)=x^{-\frac{q-1}{2}} t^{-\frac{2 p+q-3}{2}} \Delta_{L_{p, q}}(x, t),
$$

where $\Delta_{L_{p, q}}(x, t)$ is presented as in Lemma 6 The rest of the proof is an easy exercise which we shall leave to the reader.

Corollary 8. For fixed $q \geq 3$ and $n \geq 1$, the 4-manifolds $\left\{E(n, 1)_{L_{p, q}}\right\}_{p \geq 2}$ are mutually non-diffeomorphic. 
Proof. We note that the total number of the $S W$ basic classes are invariant under any diffeomorphism. As a consequence of Lemma 7 , the total number of basic classes of our 4-manifolds depend on $p$. In fact, for $q \geq 3$ and $p \geq 2$, the total number of basic classes of $E(n, 1)_{L_{p, q}}$ is $(2 n+2 q-6) p+(q n-4 n-4 q+12)$. One could check the validity of this formula by the following elementary argument which occupies the rest of the proof.

We first recall that the homology classes $\xi$ and $\tau$ are linearly independent in $H_{2}\left(E(n, r)_{L_{p, q}}\right)$ by Proposition 3.2 in [MT]. Let $N_{n, p, q}$ be the number of basic classes of $E(n, 1)_{L_{p, q}}$. Then $N_{n, p, q}$ is the number of non-zero coefficients of

$$
\begin{aligned}
\overline{S W}_{E(n, 1)_{L_{p}, q}} & =\left[\sum_{k=0}^{n-1}(-1)^{k}\left(\begin{array}{c}
n-1 \\
k
\end{array}\right) \xi^{n-2 k-1}\right]\left[\xi^{-(q-1)} \tau^{-(2 p+q-3)}+\xi^{q-1} \tau^{2 p+q-3}\right. \\
& \left.+\left(\tau^{-(2 p-4)} \sum_{i=0}^{2 p-4}\left(-\tau^{2}\right)^{i}\right)\left(\sum_{j=0}^{q-3}(\xi \tau)^{-(q-3)+2 j}\right)\right] \\
& =\left[\sum_{k=0}^{n-1}(-1)^{k}\left(\begin{array}{c}
n-1 \\
k
\end{array}\right)\left(\xi^{n-2 k-q} \tau^{3-2 p-q}+\xi^{n+q-2 k-2} \tau^{2 p+q-3}\right)\right] \\
& +\left[\sum_{k=0}^{n-1} \sum_{i=0}^{2 p-4} \sum_{j=0}^{q-3}(-1)^{i+k}\left(\begin{array}{c}
n-1 \\
k
\end{array}\right) \xi^{n+2 j+2-2 k-q} \tau^{2 i+2 j+7-2 p-q}\right] .
\end{aligned}
$$

Note that, if $n+2 j+2-2 k-q=n+2 j^{\prime}+2-2 k^{\prime}-q$ and $2 i+2 j+7-2 p-q=$ $2 i^{\prime}+2 j^{\prime}+7-2 p-q$, then $i+k=i^{\prime}+k^{\prime}$; hence $N_{n, p, q}-2 n$ is equal to the number of elements in the set

$$
\Lambda:=\{(j-k, i+j) \mid 0 \leq i \leq 2 p-4,0 \leq j \leq q-3,0 \leq k \leq n-1\} .
$$

On the other hand, the number of elements in $\Lambda$ is

$$
\# \Lambda=((2 p-4)+1)((q-3)+1)((n-1)+1)-(2 p-4)(q-3)(n-1) .
$$

To see this, consider the linear map $\rho: \mathbb{R}^{3} \rightarrow \mathbb{R}^{2}$ given by $\rho(x, y, z)=(y-z, x+y)$. The kernel of $\rho$ is the line generated by the vector $(-1,1,1)$. Hence $\# \Lambda$ is equal to the number of lines in $\mathbb{R}^{3}$ that are parallel to the vector $(-1,1,1)$ and meet an integer point in the rectangular parallelepiped

$$
\mathcal{P}:=\{(x, y, z) \mid 0 \leq x \leq 2 p-4,0 \leq y \leq q-3,0 \leq z \leq n-1\} .
$$

We see easily that the set of such lines is parametrized by the integer points on the three sides of $\mathcal{P}$ lying on the $x y$-plane, $x z$-plane, and the plane $x=2 p-4$. The number of integer points on these three sides of $\mathcal{P}$ is equal to the total number of integer points in $\mathcal{P}$ minus the number of integer points in a smaller parallelepiped of dimensions one less. See Figure 3

This proves the formula for $\# \Lambda$. Now an easy calculation gives

$$
N_{n, p, q}=(2 n+2 q-6) p+(q n-4 n-4 q+12) .
$$

It follows that the symplectic tori $\left\{T_{p, q}\right\}_{p \geq 2}$ are non-isotopic in $E(n)$, which completes the proof of Theorem 1 for $q \geq 3$. We should note that since the number of basic classes of $E(n, 1)_{L_{1, q}}$ is $q n$, which is the same as the number of basic classes of $E(n, 1)_{L_{2, q}}$, the above argument cannot be used to conclude that no torus in $\left\{T_{p, q}\right\}_{p \geq 2}$ is isotopic to $T_{1, q}$. It is also insufficient to show that the 4-manifolds $E(n, 1)_{L_{p, 2}}$ are mutually non-diffeomorphic, since the number of their basic classes 


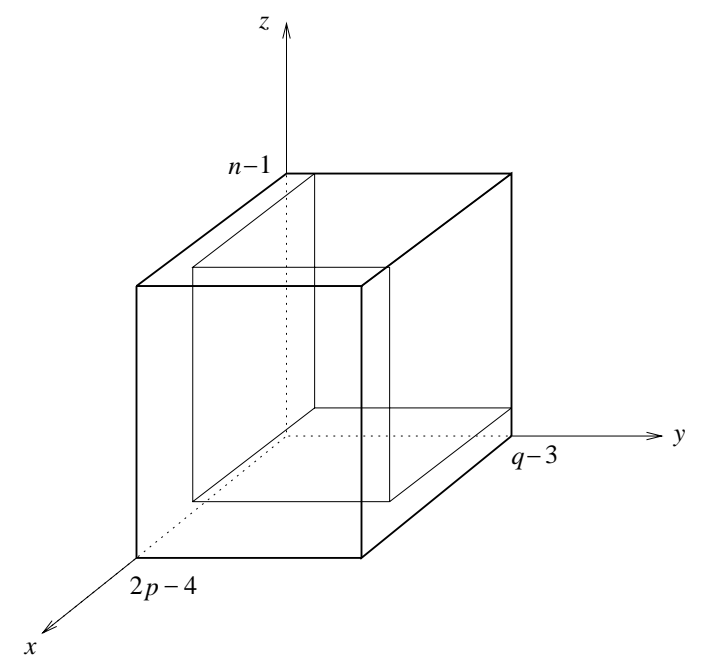

FiguRE 3 . The rectangular parallelepiped $\mathcal{P}$ in $\mathbb{R}^{3}$

are independent of $p$. Nevertheless, it is possible to prove the following corollary by comparing the divisibilities of certain basic classes of $E(n, r)_{L_{p, 2}}$.

Corollary 9. There are infinitely many mutually non-isotopic tori in the set of symplectic tori $\left\{T_{p, 2}\right\}_{p \geq 2}$ in $E(n)$.

Proof. First note that by an easy Mayer-Vietoris argument we can extend the set $\{\xi, \tau\}$ to an integral basis of $H_{2}\left(E(n, r)_{L_{p, q}} ; \mathbb{Z}\right)$ (see [GS], pp. 72-74, for the description of a basis of $\left.H_{2}([E(n) \backslash \nu F] ; \mathbb{Z})\right)$. To distinguish $T_{p_{1}, 2}$ and $T_{p_{2}, 2}$ in $E(n)$ we could choose a suitable $r$ and compare the divisibilities of basic classes with Seiberg-Witten invariant \pm 1 for $E(n, r)_{L_{p_{i}, 2}}$. When $n$ is odd and $p_{1}>p_{2}>\frac{n-1}{2}$, a comparison of the divisibilities of basic classes for $E\left(n, \frac{2 p_{1}+1-n}{2}\right)_{L_{p_{i}, 2}}$ shows that these 4-manifolds are not diffeomorphic and hence $T_{p_{1}, 2}$ and $T_{p_{2}, 2}$ are non-isotopic in $E(n)$. In case $n$ is even $r$ could be chosen as $2 p_{1}-\frac{n}{2}$ to distinguish $T_{p_{1}, 2}$ and $T_{p_{2}, 2}$ in $E(n)$ for sufficiently large $p_{2}<p_{1}$.

Remark 10. Strictly speaking, when $r \geq 2$, the diffeomorphism type of the fiber sum $E(n) \#_{T_{p, q}=F} E(r)=E(n, r)_{L_{p, q}}$ may depend not just on the isotopy class of $T_{p, q}$ but also on the chosen framing of $T_{p, q}$ that trivializes $\nu T_{p, q}$. However, given two inequivalent framings of $T_{p, q}$ in $E(n)$, the resulting two possibly non-diffeomorphic fiber sums have equivalent Seiberg-Witten invariant (given by Lemma 71). Fortunately for our purposes, the Seiberg-Witten invariant is not delicate enough to display the dependence of the diffeomorphism type of this particular fiber sum on the framing of $T_{p, q}$. Hence the collection of Seiberg-Witten invariants (in particular, the divisibilities of the basic classes) of the fiber sums is indeed an isotopy invariant of the torus $T_{p, q}$, even though the diffeomorphism types of $\left\{E(n) \#_{T_{p, q}=F} E(r)\right\}_{r \geq 2}$ by themselves may not be one.

\section{Generalization to other 4-Manifolds}

It is not hard to show that our family $\left\{T_{p, q}\right\}$ embed non-isotopically into a more general class of 4 -manifolds than $E(n)$. One way to generalize Theorem 1 is to 
use the formulas in [FS2 and show that there is an infinite family of non-isotopic symplectic tori in $q[F]$ for $q \geq 3$ provided that $F$ is a $c$-embedded symplectic torus of self-intersection 0 in a symplectic 4-manifold (cf. Theorem 5.2 in [FS3]). Another way is to utilize the product formulas for Seiberg-Witten invariants given in $\mathrm{Pa}$ ] and [T2 to generalize Theorem[1 to an even larger class of 4-manifolds. The latter will be our approach here. In the rest of this section let $F$ denote a 2-torus of self-intersection 0 , which is a symplectic submanifold of a symplectic 4-manifold $X$. We shall always assume that $[F] \in H_{2}(X ; \mathbb{Z})$ is a primitive class, i.e., $[F] \neq m \gamma$ for some $\gamma \in H_{2}(X ; \mathbb{Z})$ and $|m|>1$. Just as before, we may identify a tubular neighborhood $\nu F$ with $\left[S^{1} \times\left(S^{3} \backslash \nu A\right)\right]$, and we immediately obtain an embedding of the tori $\left\{T_{p, q}\right\}$ into $\nu F \subset X$. It is easy to check that Lemma 3 continues to hold in this more general setting, i.e., $T_{p, q} \subset X$ is a symplectic submanifold representing the homology class $q[F]$.

To show that the family $\left\{T_{p, q}\right\}$ are mutually non-isotopic in $X$, we need to again show that the corresponding 4-manifolds,

$$
\begin{gathered}
X \#_{\varphi_{p, q}} E(r):=\left[X \backslash \nu T_{p, q}\right] \bigcup_{T_{p, q} \times \partial D^{2}=F_{2} \times \partial D^{2}}\left[E(r) \backslash \nu F_{2}\right] \\
=[X \backslash \nu F] \bigcup_{F \times \partial D^{2}=\left(S^{1} \times \alpha_{1}\right) \times \beta_{1}}\left[S^{1} \times\left(S^{3} \backslash \nu L_{p, q}\right)\right] \\
\bigcup_{\left(S^{1} \times \alpha_{2}\right) \times \beta_{2}=F_{2} \times \partial D^{2}}\left[E(r) \backslash \nu F_{2}\right],
\end{gathered}
$$

are mutually non-diffeomorphic. Here we again choose the homology basis $\left(\alpha_{1}, \beta_{1}\right)$ $=(\mu(A), \lambda(A))$, and $\left(\alpha_{2}, \beta_{2}\right)=\left(\lambda\left(\hat{B}_{p, q}\right),-\mu\left(\hat{B}_{p, q}\right)\right)$ when we do link surgery. To compute the Seiberg-Witten invariants, we will need the following definition.

Definition 11. A surface $F \subset X$ is said to be essentially embedded if there exists a homology class $\delta \in H_{2}(X ; \mathbb{Z})$ such that $[F] \cdot \delta \neq 0$.

For example, a torus fiber $F_{2} \subset E(r)$ is essentially embedded since we can take $\delta$ to be the homology class of a section. Note that a surface $F$ is essentially embedded in a 4-manifold $X$ if and only if its homology class $[F] \in H_{2}(X ; \mathbb{Z})$ is a non-torsion element. Also note that a symplectic surface $F$ in a symplectic 4 -manifold is always essentially embedded.

Lemma 12. Suppose $F \subset X$ is an essentially embedded 2-torus of self-intersection 0. If $H^{1}(X \backslash \nu F ; \mathbb{Z})=0$, then

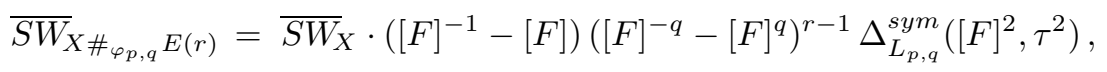

where $\tau:=\iota_{*}\left[S^{1} \times \mu\left(\hat{B}_{p, q}\right)\right] \in H_{2}\left(X \#_{\varphi_{p, q}} E(r) ; \mathbb{Z}\right)$ is defined as in Lemma $\square$

Proof. Corollary 20 in $[\mathrm{Pa}]$ gives

$$
\overline{S W}_{X \backslash \nu F}=\overline{S W}_{X} \cdot\left([F]^{-1}-[F]\right) .
$$

The rest of the proof goes exactly the same way as in the proof of Lemma 7 Again we need to identify $\left[F_{2}\right]=q[F]$ for the same reasons as in the proof of Lemma 7

Corollary 13. Suppose that $F$ is a symplectic 2-torus in a symplectic 4-manifold $X$ with $b_{2}^{+}(X)>1$. Also assume that $[F] \in H_{2}(X ; \mathbb{Z})$ is primitive, $[F] \cdot[F]=0$, and $H^{1}(X \backslash \nu F ; \mathbb{Z})=0$. Then for fixed $q \geq 3$, there are infinitely many manifolds in the set of symplectic 4-manifolds $\left\{X \#_{\varphi_{p, q}} E(1)\right\}_{p \geq 2}$ that are mutually non-diffeomorphic. 
Proof. When $b_{2}^{+}(X)>1$, we always have $\overline{S W}_{X} \neq 0$ by Taubes' result [T1]. Note that the homology classes $[F]$ and $\tau$ are linearly independent in $H_{2}\left(X \#_{\varphi_{p, q}} E(1)\right)$ by Proposition 3.2 in [MT. The rest of the proof is an easier analogue of the proof of Corollary 8 . We formally set $\tau=[F]$, and note that as $p \rightarrow \infty$, the total number of terms in

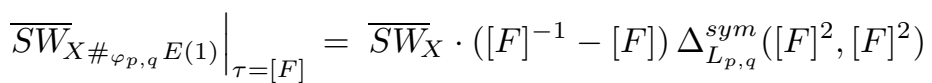

goes to $\infty$ as well. This immediately implies that the number of $S W$ basic classes of $X \#_{\varphi_{p, q}} E(1)$ also goes to $\infty$ as $p \rightarrow \infty$.

Now we can readily generalize Theorem 1 to other symplectic pairs $(X, F)$ as follows.

Theorem 14. Suppose that $F$ is a symplectic 2-torus in a symplectic 4-manifold $X$ with $b_{2}^{+}(X)>1$. Also assume that $[F] \in H_{2}(X ; \mathbb{Z})$ is primitive, $[F] \cdot[F]=0$, and $H^{1}(X \backslash \nu F ; \mathbb{Z})=0$. Then for any integer $q \geq 3$, there exists an infinite family of mutually non-isotopic symplectic tori representing the homology class $q[F]$.

Note that, Corollary 9 cannot be easily generalized, since the divisibilities of basic classes of $X \#_{\varphi_{p, q}} E(r)$ depend heavily on $\overline{S W}_{X}$ and $H_{2}(X ; \mathbb{Z})$.

Remark 15. One must take care and define $\overline{S W}_{X}:=\overline{S W}_{X, F}^{ \pm}$when $b_{2}^{+}(X)=1$ (see [FS2 and [Pa]). When $b_{2}^{+}(X)=1$, it is not automatic that $\overline{S W}_{X}$ is a finite sum and $\overline{S W}_{X} \neq 0$ for a symplectic $X$. If indeed $\overline{S W}_{X} \neq 0$ and is a finite sum, then Theorem 14 will still be valid for such a pair $(X, F)$. However, if $\overline{S W}_{X}=0$ or is an infinite sum, then there seems to be no method currently available to check whether the tori in our family are mutually non-isotopic in $X$. Along this line, it has been conjectured that an infinite family of homologous but non-isotopic symplectic tori cannot occur in $X$, when $X$ is a rational ruled surface with $c_{1}^{2}(X)>0$. In fact, Sikorav has already proved this conjecture for $X=\mathbb{C P}^{2}$ in [Si] and Siebert and Tian announced a proof for $X=S^{2} \times S^{2}$ and $X=\mathbb{C P}^{2} \# \overline{\mathbb{C P}}^{2}$ (see [ST1]).

Remark 16. We should also point out that since our proof uses the product formula to calculate the Seiberg-Witten invariants of 4-manifolds obtained by gluing along the boundary of a regular neighborhood of a torus and since such product formulas do not give the complete picture in case the gluing occurs along higher genus surfaces, we were able to construct infinite families of non-isotopic tori only. In fact, there is an ongoing research to determine the number of symplectic representatives (up to smooth isotopy) in the homology class of symplectic surfaces of higher genus. For example in $[\mathrm{Sm}$, by using purely topological techniques, Smith was able to prove that for every odd number $g \neq 3$ there exists a symplectic 4-manifold $X_{g}$ that contains an infinite family of homologous but non-isotopic connected symplectic surfaces of genus $g$. On the other hand, Siebert and Tian announced in ST2 that there is a unique symplectic surface in the homology class of a degree $d$ complex curve in $\mathbb{C P}^{2}$ provided that $d \leq 17$ and similarly in the homology class of a complex curve of bidegree $(m, n)$ in a Hirzebruch surface provided that $m \leq 7$.

\section{ACKNowledgments}

The second author would like to thank Ronald Fintushel and Stefano Vidussi for helpful discussions. Some special cases of the computations in Section 3 were verified with the aid of Maple ${ }^{\circledR}$ Version 8. 


\section{REFERENCES}

[BZ] G. Burde and H. Zieschang: Knots, de Gruyter Studies in Mathematics 5, Walter de Gruyter, Berlin, New York, 1985. MR 87b:57004

[FS1] R. Fintushel and R.J. Stern: Rational blowdowns of smooth 4-manifolds, J. Differential Geom. 46 (1997), 181-235. MR 98j:57047

[FS2] R. Fintushel and R.J. Stern: Knots, links and 4-manifolds, Invent. Math. 134 (1998), 363-400. MR 99j:57033

[FS3] R. Fintushel and R.J. Stern: Symplectic surfaces in a fixed homology class, J. Differential Geom. 52 (1999), 203-222. MR 2001j:57036

[GS] R.E. Gompf and A.I. Stipsicz: 4-Manifolds and Kirby Calculus, Graduate Studies in Mathematics 20, Amer. Math. Soc., 1999. MR 2000h:57038

[Ma] T. Matumoto: Extension problem of diffeomorphisms of a 3-torus over some 4-manifolds, Hiroshima Math. J. 14 (1984), 189-201. MR 86b:57016

[MT] C.T. McMullen and C.H. Taubes: 4-manifolds with inequivalent symplectic forms and 3-manifolds with inequivalent fibrations, Math. Res. Lett. 6 (1999), 681-696. MR 2000m:57045

[Mo] H.R. Morton: The multivariable Alexander polynomial for a closed braid, in Lowdimensional Topology, ed. Hanna Nencka, Contemporary Mathematics 233, Amer. Math. Soc. (1999), 167-172. Also available at arXiv:math.GT/9803138. MR 2000e:57025

[Pa] B.D. Park: A gluing formula for the Seiberg-Witten invariant along $T^{3}$, Michigan Math. J. 50 (2002), 593-611. MR 2003i:57051

[Ro] D. Rolfsen: Knots and Links, Publish or Perish Inc., Houston, 1990; Corrected reprint of the 1976 original. MR 95c:57018

[ST1] B. Siebert and G. Tian: On hyperelliptic $C^{\infty}$-Lefschetz fibrations of four-manifolds, Commun. Contemp. Math. 1 (1999), 255-280. Also available at arXiv:math.GT/9903006. MR 2001g:57053

[ST2] B. Siebert and G. Tian: Tian's talk at the Conference on Holomorphic Curves and LowDimensional Topology, Institute for Advanced Study, March 2002.

[Si] J-C. Sikorav: The gluing construction for normally generic $J$-holomorphic curves, Symplectic and contact topology: interactions and perspectives, Fields Inst. Commun., 35, pp. 175199, Amer. Math. Soc., Providence, RI, 2003. Also available at arXiv:math.SG/0102004.

[Sm] I. Smith: Symplectic submanifolds from surface fibrations, Pacific J. Math. 198 (2001), 197-205. MR 2002b:57029

[T1] C.H. Taubes: The Seiberg-Witten invariants and symplectic forms, Math. Res. Lett. 1 (1994), 809-822.

[T2] C.H. Taubes: The Seiberg-Witten invariants and 4-manifolds with essential tori, Geom. Topol. 5 (2001), 441-519. MR 2002d:57025

[V1] S. Vidussi: Smooth structure of some symplectic surfaces, Michigan Math. J. 49 (2001), 325-330. MR 2002f:57057

[V2] S. Vidussi: Nonisotopic symplectic tori in the fiber class of elliptic surfaces, preprint. Available at http://www.math.ksu.edu/ ${ }^{\sim}$ vidussi/

Department of Mathematics and Statistics, McMaster University, Hamilton, OnTARIO, CANADA L8S $4 \mathrm{~K} 1$

E-mail address: etgut@math.mcmaster.ca

Department of Pure Mathematics, University of Waterloo, Waterloo, Ontario, CanADA N2L 3G1

E-mail address: bdpark@math.uwaterloo.ca 